Check for updates

Cite this: RSC Adv., 2017, 7, 27595

\title{
Digital immunoassay of a prostate-specific antigen using gold nanorods and magnetic nanoparticles $\uparrow$
}

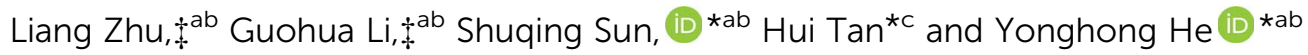

Herein, a digital immunoassay was developed by counting highly confined gold nanorod (AuNR) probes with the aid of magnetic nanoparticles (MNPs). MNPs were conjugated with capture antibodies to concentrate the target analytes. AuNRs were conjugated with a mixture of the detection antibody and randomly selected DNA, where the detection antibody was applied to signal the target binding event and DNA was applied to provide sufficient negative charges. The bicomponent AuNR probes in the targetmediated immunocomplexes were eluted and confined onto a positively charged glass slide on the basis of electrostatic attraction for accurate counting. The combination of the magnetic concentration of the target analytes and the confinement of the DNA/antibody AuNR probes results in improved detection efficiency; a good linear range from 0.01 to $10 \mathrm{pg} \mathrm{mL}^{-1}$ and a detection limit of $8 \mathrm{fg} \mathrm{mL}^{-1}$ ( 226 aM) were achieved for the detection of prostate-specific antigen (PSA). The proposed method offers the simultaneous advantages of high sensitivity, digital quantification accuracy, lack of requirement for signal or target amplification, wide dose response curve and low detection cost; thus, it shows good potential in practical applications.
\end{abstract}

Received 14th January 2017

Accepted 26th March 2017

DOI: $10.1039 / c 7 r a 00575 j$

rsc.li/rsc-advances and a high-intensity fluorophore reporter. Typical optical systems involve confocal microscopy, flow-based ultrasensitive single-molecule counting systems ${ }^{8}$ or total internal reflection fluorescence microscopy (TIRFM). ${ }^{\mathbf{9}, \mathbf{1 0}}$ These systems often require high-efficiency collection optics, excellent detectors with high quantum efficiency and low dark noise, prebleaching of impurities and specific facilities to reduce background. Therefore, all these will significantly increase the operational complexity and instrument cost, limiting the practical applications of SMD methods. Fluorophore reporters include organic dyes, ${ }^{9,11,12}$ quantum dots (Qds), ${ }^{10,13,14}$ and fluorescent nanoparticles. ${ }^{15-17}$ However, fluorescent organic dyes are limited by low absorption cross sections, ${ }^{18}$ photobleaching ${ }^{19}$ and photoquenching: ${ }^{20}$ Qds demonstrate the drawbacks of photoblinking and biological toxicity. ${ }^{21}$ The inherent defects of the fluorophore reporters result in inaccurate detection results; therefore, they represent an important limit to SMD methods.

Gold nanorods (AuNRs) with effective sizes ranging from 10s to 100 s of nanometers are plasmonic nanoparticles that have gained much attention because of their extraordinary localized surface plasmon resonance (LSPR) properties. ${ }^{22}$ A remarkable property of AuNRs is extremely strong selective scattering in the visible region based on their sizes, components and shapes. Typically, the scattering intensity of an individual AuNR may be several orders of magnitude higher than that of a strong fluorescent dye. ${ }^{23}$ Thus, AuNRs are easily and unambiguously identified from the background using cost-effective and easyattainable dark-field microscopy. In addition, AuNRs are readily distinguished from impurity particles because of their
Invasive Medical Imaging and Sensing, Shenzhen Key Laboratory for Minimal Shenzhen 518055, People's Republic of China. E-mail: sun.shuqing@sz.tsinghua. edu.cn; heyh@sz.tsinghua.edu.cn

${ }^{b}$ Department of Physics, Tsinghua University, Beijing 100084, People's Republic of China

${ }^{c}$ The First Affiliated Hospital of Shenzhen University, Shenzhen Key Laboratory of Neurosurgery, Shenzhen, 518035, China.E-mail: tanhui@iccas.ac.cn

$\dagger$ Electronic supplementary information (ESI) available: Details experimental section and additional experimental results. See DOI: 10.1039/c7ra00575j

\$ These two authors contribute equally. 
specific scattering spectrum characterization. These excellent optical properties have enabled many promising applications, such as analytical chemistry, cell imaging, ${ }^{24}$ photothermal therapy ${ }^{25}$ and real-time monitoring of chemical reactions at the single-particle level. ${ }^{26}$ In addition, AuNRs have excellent optical and chemical stability, which is another significant advantage over conventional fluorescence reagents. However, even with these remarkable advantages in their optical properties and detection costs, there are few reports on the application of AuNRs in SMD-based quantitative analysis.

Magnetic beads have gained widespread traction due to their superior performance in the separation and purification of biomolecules. ${ }^{27,28}$ The main merit of the utilization of magnetic nanoparticles is that the magnetic separation process can be rapidly performed using only a common magnet without the requirement of expensive instrumentation. This simplicity is not available for conventional separation methods, such as centrifugation and bead-packed columns. An interesting feature of magnetic beads is that they can be functionalized with specific biomolecules for DNA hybridization and immunoassays.

Herein, we report on the proof of concept of a novel digital immunoassay based on single-countable AuNRs and magnetic nanoparticles (MNPs) (Fig. 1). Prostate-specific antigen (PSA) was chosen in this study as the initial target because it is an important indicator for prostate cancer, which is one of the most common diseases in elderly men. MNPs are modified with monoclonal antibodies and AuNRs are modified with a mixture of polyclonal antibodies and randomly selected DNA sequences. In the presence of the target, AuNRs and MNP sandwich the PSA target, forming a PSA-mediated immunocomplex because of the target-specific binding. After removing the excess unbound AuNRs, the binding interactions are disrupted by the addition of elution buffer. Each PSA molecule in the sample solution corresponds to a released AuNR in the elution buffer. Therefore, a positive correlation exists between the target concentration and the number of AuNRs in the elution buffer. The sensitivity to detect PSA is directly related to the sensitivity to detect AuNRs. To enumerate the AuNRs more effectively, the AuNRs are modified with randomly selected DNA sequences to provide sufficient negative charges arising from the phosphate backbone of DNA. This treatment enables the AuNRs to be easily immobilized and subsequently highly confined onto a positively charged APTES-modified glass slide via electrostatic attraction. Simply counting the AuNRs in the confined ensures a relatively high sampling rate. Combined with the single particle identification, an extremely low concentration of AuNRs can also be detected.

\section{Materials and methods}

\section{Materials and instruments}

Carboxyl-modified magnetic nanoparticles were provided by Enriching Biotechnology Ltd (China). Monoclonal antibody to prostate-specific antigen was purchased from Exbio (11-289C100, Czech Republic). Rabbit polyclonal to human prostatespecific antigen was purchased from GeneTex. $\mathrm{HAuCl}_{4} \cdot 3 \mathrm{H}_{2} \mathrm{O}$, $\mathrm{NaBH}_{4}, \mathrm{AgNO}_{3}$, cetyltrimethylammonium bromide (CTAB), prostate-specific antigen (P3235), Traut's reagent, tris(2carboxyethyl)phosphine (TCEP), Tris-HCl, bovine serum albumin (BSA), sodium citrate, 2 -( $N$-morpholino)ethanesulfonic acid (MES) and polyvinylpyrrolidone (PVP, M.W. 8000) were purchased from Sigma-Aldrich. 1-Ethyl-3-(3dimethylaminopropyl) carbodiimide hydrochloride (EDC) was purchased from the Tokyo Kasei Kogyo Co. (Japan); working solutions were prepared with ice-cold ultrapure water immediately before use. The nucleic acid sequence (5'-GTG TGG ATA ATA GAG $\left.\mathrm{A}_{10}-\left(\mathrm{CH}_{2}\right)_{6}-\mathrm{SH}-3^{\prime}\right)$ was purchased from Beijing Genomics Institution (China). A dark field microscope (BX53, Olympus, Japan) equipped with a color CCD camera (DP73, Olympus, Japan) and a $150 \mathrm{~W}$ halogen lamp was used to obtain images of the AuNRs. The extinction spectrum was measured by a UV-Vis-NIR spectrophotometer (Cary 5000, Varian, USA). The particle size distributions were measured by a Zetasizer Nano ZS

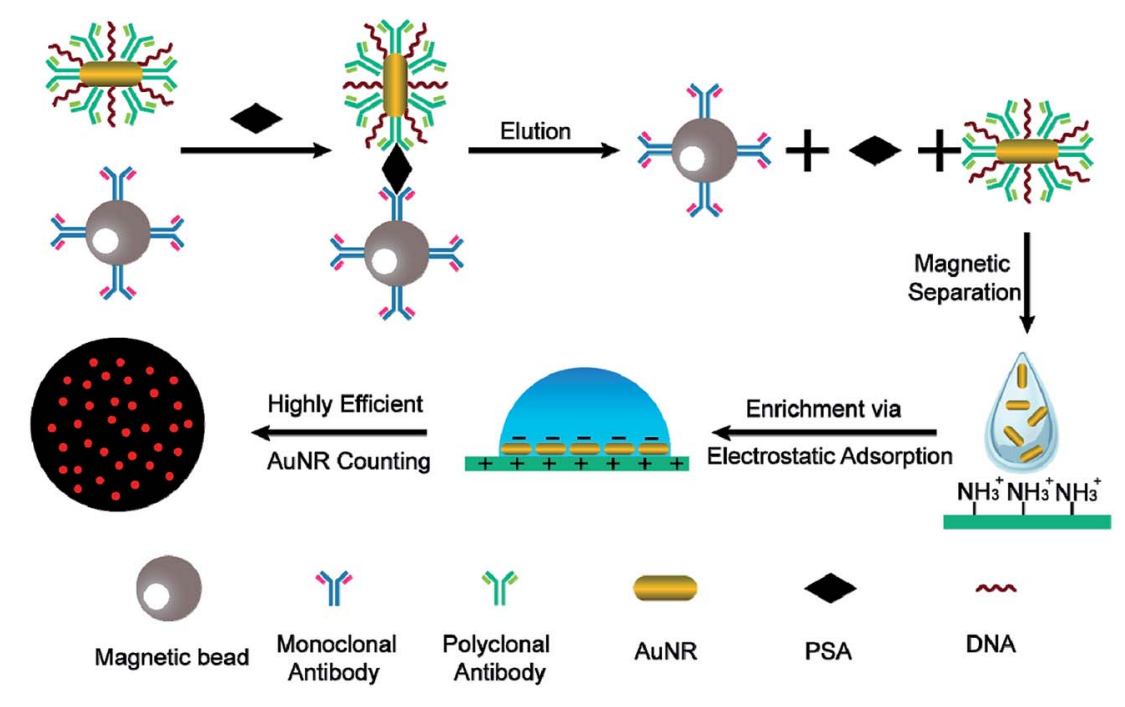

Fig. 1 Schematic of the detection of PSA. 
system (Malven, U.K.). The gold content was measured by inductively coupled plasma-atomic emission spectrometry (ICPAES, JY2000-2, Horiba, France).

\section{Conjugation of the monoclonal antibody to MNPs}

Magnetic nanoparticles (MNPs, $200 \mathrm{~nm}$ ) were functionalized with monoclonal antibody according to the manufacturer's instructions. $50 \mu \mathrm{L}$ of $10 \mathrm{mg} \mathrm{mL} \mathrm{m}^{-1}$ MNPs were washed twice with $50 \mathrm{mM}$ pH 5.1 MES buffer containing $0.03 \%$ SDS. $50 \mu \mathrm{L}$ of MES buffer and $10 \mu \mathrm{L}$ of freshly prepared $10 \mathrm{mg} \mathrm{mL}^{-1}$ cold EDC prepared in MES buffer were added and were mixed well by vortex. The MNPs were resuspended and incubated for half an hour at $25{ }^{\circ} \mathrm{C}$. After the incubation, the supernatant was removed. The MNPs were collected and washed once with cold ultrapure water and twice with MES buffer as soon as possible to avoid hydrolysis of the EDC-activated carboxyl groups. The wash buffer was removed, and $5 \mu \mathrm{g}$ of monoclonal anti-PSA in $50 \mu \mathrm{L}$ of $50 \mathrm{mM}$ MES buffer was added to the activated MNPs. The activated MNPs were resuspended and incubated for at least 1 hour at $25{ }^{\circ} \mathrm{C}$ and 2 hours at $4{ }^{\circ} \mathrm{C}$. After the incubation process, the monoclonal antibody was successfully conjugated to the MNPs. A magnet was applied to collect the MNPs. The coated beads were incubated with $50 \mu \mathrm{L}$ of $50 \mathrm{mM} \mathrm{pH} 7.4$ tris buffer containing $0.1 \%$ SDS for 1 hour at room temperature to quench the non-reacted carboxyl groups. A magnet was applied to collect the MNPs, and $50 \mu \mathrm{L}$ of $1 \%$ BSA (bovine serum albumin) in $0.01 \mathrm{M}$ pH 7.4 PBS buffer was added to the pelleted beads. After incubation for 1 hour, the beads were washed four times with $100 \mu \mathrm{L}$ of PBS buffer. The obtained monoclonal antibodycoated MNPs were resuspended and stored in $1 \mathrm{~mL}$ of $\mathrm{pH} 7.4$ PBS buffer before use.

\section{Preparation of the AuNR probes}

The synthesis of the AuNRs is described in the ESI. $\dagger$ The basic principle to prepare the Ab/DNA bicomponent AuNR probes is demonstrated in Fig. S1. $\dagger$ It is well known that thiol groups have an excellent affinity to gold element. When the antibody contains a thiol group, it is much more readily immobilized onto the AuNR surface through Au-S bonds. In view of this principle, polyclonal anti-PSA was first modified with Traut's reagent in order to introduce free thiol groups into its amines (Fig. S1a $\dagger){ }^{29-31}$ In our method, an aliquot $(10 \mu \mathrm{L})$ of the polyclonal antibody solution was mixed with $10 \mu \mathrm{L}$ of $10 \mathrm{mg} \mathrm{mL}^{-1}$ Traut's reagent dissolved in $0.01 \mathrm{M}$ pH 7.4 PBS buffer. Afterwards, the mixture was incubated for 2 hours at $25{ }^{\circ} \mathrm{C}$. Unreacted Traut's reagent was removed through dialysis for about $48 \mathrm{~h}$. The obtained thiolated antibodies can then be used for further biofunctionalization.

Subsequently, the as-synthesized AuNR solution was pipetted into a $10 \mathrm{~mL}$ centrifuge tube. Excess CTAB molecules on the AuNR surfaces were removed by centrifugation at $8000 \mathrm{rpm}$ several times. The resulting pelleted particles were redispersed in $20 \mathrm{~mL}$ of PBS buffer containing $0.1 \%$ SDS and mixed well with $20 \mathrm{~mL}$ of $10 \%$ PVP solution in ethanol. The mixture was vigorously stirred for 24 hours at $42{ }^{\circ} \mathrm{C}$. CTAB molecules coated on the surface of the AuNRs were replaced by a mixture of PVP and SDS (Fig. S1b†). After a period of stirring, the resulting product was centrifuged for $15 \mathrm{~min}$ at $8000 \mathrm{rpm}$ to remove the supernatant and then redispersed in $100 \mu \mathrm{L}$ of $0.01 \mathrm{M}$ PBS buffer 3 more times. $10 \mu \mathrm{g}$ of thiol-modified polyclonal anti-PSA was added and incubated for 1 hour at room temperature. 0.2 OD (optical density) of TCEP deprotected thiol-DNA (5'-GTG TGG ATA ATA GAG $\left.\mathrm{A}_{10}-\left(\mathrm{CH}_{2}\right)_{6}-\mathrm{SH}-3^{\prime}\right)$ was added and incubated for 6 hours. In order to stabilize the products, salt solution (10 mM pH 8.0 PBS, 0.3 M NaCl, $4 \mathrm{mM} \mathrm{MgCl}_{2}, 0.2 \%$ SDS) was added in a stepwise manner to ensure the final concentration of $\mathrm{NaCl}$ was $0.15 \mathrm{M}$. This salting procedure was completed in 6 hours with a time interval of 1 hour. The resulting salted AuNR solution was left at room temperature for an additional 12 hours to maximize the loading of the thiol-modified polyclonal anti-PSA and DNA onto the AuNRs. Next, $100 \mu \mathrm{L}$ of $1 \%$ BSA solution was added followed by incubation for 1 hour to passivate the AuNRs. The products were centrifuged for $15 \mathrm{~min}$ at $8000 \mathrm{rpm}$ and redispersed in solution containing $0.01 \mathrm{M}$ PBS. This centrifugation procedure was repeated 3 more times to remove the excess unloaded thiol-modified DNA and polyclonal anti-PSA. The final obtained AuNRs were resuspended in $200 \mu \mathrm{L}$ of solution containing $0.01 \mathrm{M}$ PBS and $0.03 \%$ SDS and stored at $4{ }^{\circ} \mathrm{C}$ in a refrigerator prior to use.

\section{Detailed detection of PSA}

The confinement of the AuNR probes is described in the ESI. $\dagger$ In a typical PSA detection experiment, an aliquot $(100 \mu \mathrm{L})$ of the actual PSA sample solution and $10 \mu \mathrm{L}$ of MNP probe solution were mixed well in a $2 \mathrm{~mL}$ centrifugal tube and then incubated for 1 hour at room temperature with gentle shaking. A magnet was introduced to collect the MNPs. The collected MNPs were washed three times with PBS buffer. An aliquot $(5 \mu \mathrm{L})$ of the prepared AuNR probe solution was added, followed by incubation for 2 hours with gentle shaking. The beads were washed 6 times to remove the unbound AuNR probes using a magnet. After the last washing procedure, $10 \mu \mathrm{L}$ of $4 \mathrm{M}$ urea was added to disrupt the antibody-analyte interactions in the immunosandwich complexes. The magnet was introduced to collect the magnetic beads, and the supernatant was transferred to a $200 \mu \mathrm{L}$ centrifuge tube for quantification. To confine the AuNR probes, $1 \mu \mathrm{L}$ of the AuNR probes was deposited onto an APTESmodified glass slide. After being allowed to stand for $15 \mathrm{~min}$, a clean coverslip was gently placed onto the slide. The prepared glass slide can be directly observed under a dark field microscope.

\section{Results and discussion}

\section{Characterization}

AuNRs with an average aspect ratio of 2.1 and an average effective radius of $17.1 \mathrm{~nm}$ were synthesized. Two plasmonic resonance modes ${ }^{32}$ corresponding to the light extinction along the long axis and the short axis can be clearly seen in Fig. 2 . The longitudinal plasmonic mode can be tuned from visible to nearinfrared; this is mainly determined by its geometrical shape and the chemical properties of the surrounding medium. Changes 


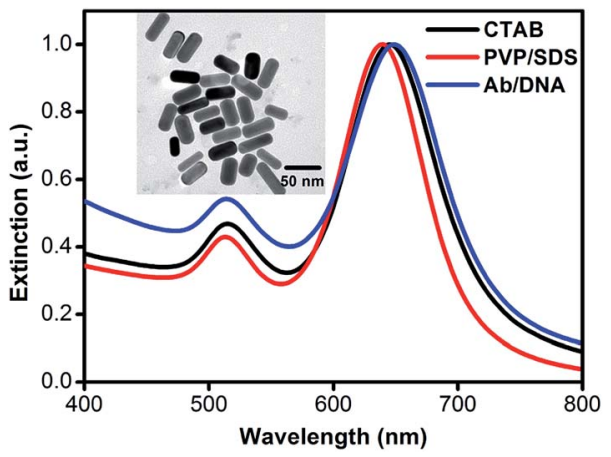

Fig. 2 UV-vis extinction spectra of AuNRs capped with CTAB, PVP/ SDS mixture and Ab/DNA mixture.

in the dielectric properties (i.e., dielectric constant or thickness) lead to peak shifts for the longitudinal modes, providing an indicator to monitor changes in the surface chemistry and the biological recognition process. The as-synthesized AuNRs are capped with CTAB bilayer to facilitate growth along the longitudinal axis and avoid aggregation. A longitudinal LSPR peak at $\sim 645 \mathrm{~nm}$ is observed. However, the CTAB bilayer is tightly packed and hinders the formation of $\mathrm{Au}-\mathrm{S}$ bonds between thiolated molecules and the AuNR surface, which is not favorable for further biofunctionalization. Moreover, many biomacromolecules, such as DNA and some proteins, are negatively charged; however, СТАВ is a typical cationic surfactant. The direct influence is that irreversible aggregation is liable to occur due to nonspecific electrostatic interactions. To prevent these problems, the as-synthesized AuNRs were repeatedly washed to remove superfluous CTAB, and the surfactant mixture of PVP/SDS was applied as an unstable intermediate stabilizing layer to replace the remaining CTAB. ${ }^{33}$
In addition, the interaction between the carbonyl on the pyrrolidone subunit and the gold atom is much weaker than the $\mathrm{Au}-\mathrm{S}$ bond. Thiolated molecules can be more easily anchored onto the AuNR surface by replacing the surfactant mixture. A blue-shift of $6 \mathrm{~nm}$ in the longitudinal LSPR peak was observed after the CTAB bilayer was exchanged with the surfactant mixture. This is because the blue-shift due to the collapse of the CTAB bilayer overcomes the red-shift due to the PVP/SDS coating. Subsequently, conjugation of AuNRs with Ab/DNA complex leads to a red-shift of $\sim 10 \mathrm{~nm}$, suggesting a significant change in the surface chemistry properties of the AuNRs. Meanwhile, the intensity ratio of the longitudinal peak to the transverse peak also decreases after Ab/DNA is conjugated onto the AuNR surfaces, which is in agreement with previous observations. $^{34}$ The transverse peak has no apparent change during the conjugation process because it is insensitive to the dielectric feature. No significant changes in the shape of the extinction spectra or peak broadening are observed, indicating that the AuNRs are not aggregated during the process of biofunctionalization. ${ }^{35-37}$

The successful biofunctionalization process was further confirmed by dynamic light scattering (DLS, Fig. S2 $\dagger$ ), which is a powerful tool to measure particle size distribution. ${ }^{36,38-40}$ The average hydrodynamic diameter of the AuNRs shows little change after the surfactant exchange process. However, an average increase of about $25 \mathrm{~nm}$ was observed after the Ab/DNA complex was conjugated onto AuNRs, also indicating the successful conjugation process.

\section{Confirmation of the biological functionality of the AuNR probes}

To confirm that the thiolated antibodies still have the same biological functionality to recognize PSA, the sandwich
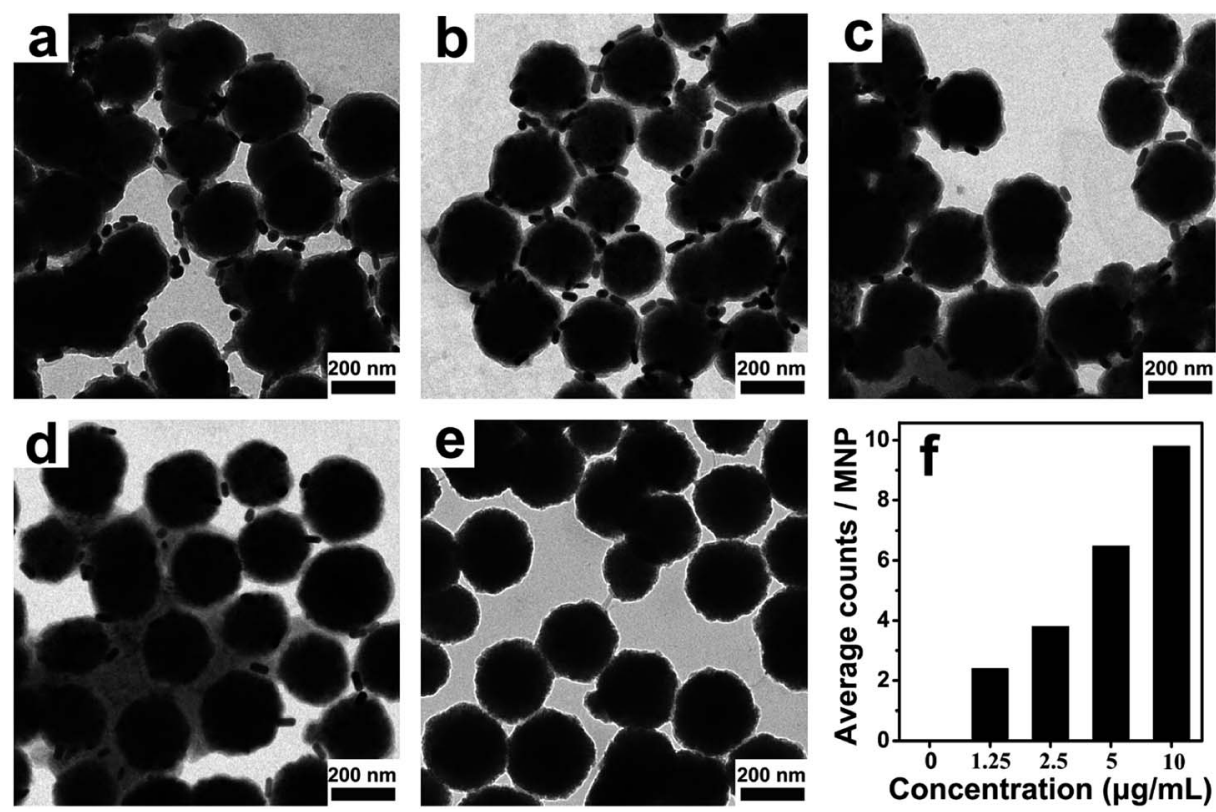

Fig. 3 Representative SEM images of the core-satellite structure of MNP/AuNR in the presence of PSA with concentrations of (a) 10, (b) 5, (c) 2.5, (d) 1.25 and (e) $0 \mu \mathrm{g} \mathrm{mL}^{-1}$. (f) The average numbers of AuNRs on a single MNP at different PSA concentrations. 
immunocomplexes obtained by the PSA-mediated two-step immunoreactions were imaged by transmission electron microscope (TEM). Unreacted AuNR probes were removed to eliminate potential interference with the experiment results. As can be seen in Fig. 3, most of the MNPs are surrounded by a few AuNRs in the presence of PSA. In contrast, few AuNR probes are seen on the surface of the MNPs in the control experiment where PSA is absent. With increasing PSA concentration, the average number of AuNRs on a single MNP also increased (statistically analyzed from more than 50 MNPs for each concentration), indicating good biological functionality and specificity. The results presented here demonstrated that PSA can be sandwiched by the AuNRs and MMP via target binding events, providing the basis for further quantitative measurements.

\section{Confinement of the AuNR probes}

Gold nanoparticles can be immobilized onto an APTES-treated glass slide via electrostatic interactions. ${ }^{\mathbf{4 1 , 4 2}}$ A self-assembled monolayer of gold nanoparticles can thus be simply obtained and has been utilized for real-time monitoring of the dynamic processes of the binding kinetics of biomolecules ${ }^{43}$ and give good quantitative results for biomolecule sensing. ${ }^{44}$ In this work, the APTES-based processing method is used to confine gold nanoparticles to ensure relatively high detection efficiency. In addition to single-particle imaging, extremely low concentrations of AuNR can be detected. To qualitatively demonstrate the confinement effect, a $10 \times$ objective was used to obtain dark field images. Considering the limited field of view (FOV) of a single dark field image, it is difficult to demonstrate the distribution of the AuNRs and the confinement effect. $1 \mu \mathrm{L}$ of AuNR probe solution was imaged, and 4 continuous images were obtained to provide the complete picture. After using a cross-correlation algorithm to remove the overlaps between two adjacent images, a reconstructed image was obtained. This reconstructed image clearly reveals the distribution of AuNR on the glass slide surface. As is shown in Fig. 4a, the high-intensity red AuNRs are highly concentrated in a perfect circular region. Meanwhile, very few red dots can be observed outside of this region. The enormous contrast between the inside and outside of the circular region demonstrates the excellent confinement performance. Additional images with higher magnification $(40 \times)$ to show the confinement effects near the boundary are displayed in Fig. S3. $\uparrow$ Therefore, simply counting the confined AuNR probes is sufficient to ensure a high sampling rate and thus a high quantification accuracy. As a comparison, the confinement effect was not as obvious for the polyclonal antibody-conjugated AuNRs (prepared with the same experimental conditions as the AuNR probes except for the addition of DNA).

An objective with smaller magnification affords a much greater FOV; however, the quality of the captured images is much poorer. To obtain clearer single AuNR images for higher accuracy in the quantification of AuNRs, a $40 \times$ objective was used instead of a $10 \times$ objective to image the AuNRs. An inhouse-coded program to recognize and count AuNRs was used to reduce labour costs and eliminate the impurity particles as much as possible. Typical recognition results are shown in Fig. S4. $\dagger$ Quantitative results for the detection of the AuNR probes were achieved by counting the red spots in the circular region in a series of concentrations. A calibration curve with a slope of 0.98 and an excellent correlation coefficient of $R^{2}=$ 0.998 was obtained within the range from 10 aM to $100 \mathrm{fM}$ (Fig. 4b). Most ( $>90 \%$ in the range) of the AuNRs are confined by the glass slide for highly efficient detection, showing good quantitative ability for highly sensitive AuNR detection. Much

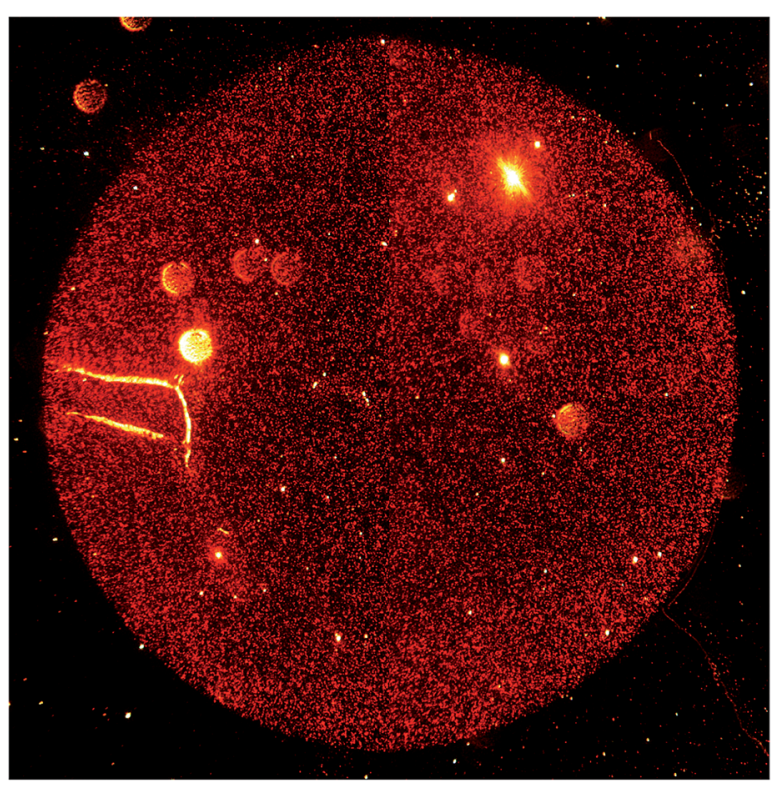

(a)

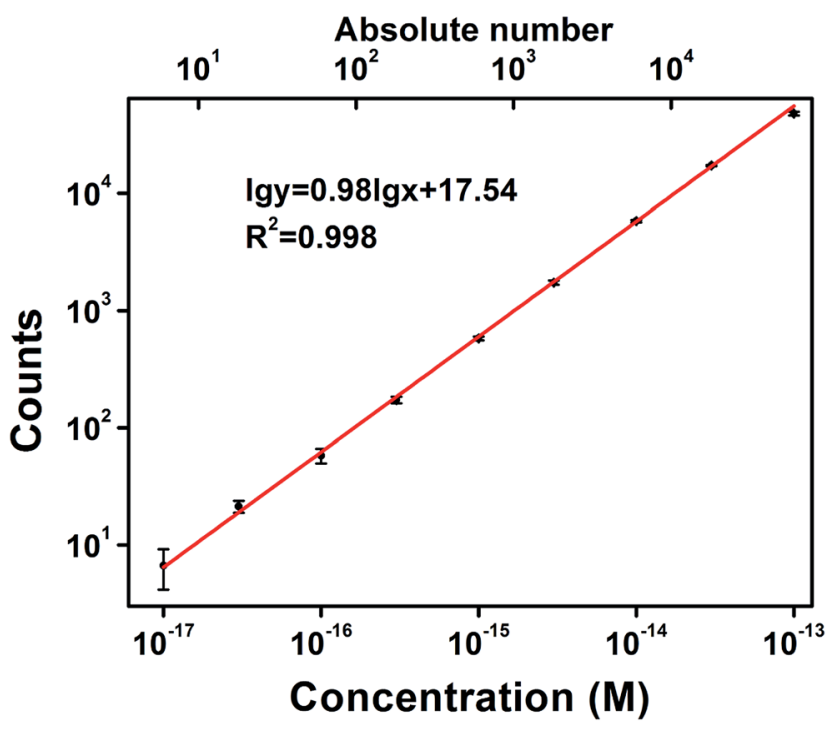

(b)

Fig. 4 (a) Typical dark field image of the AuNR probes at a concentration of $0.5 \mathrm{pM}$, reconstructed from 4 single images. The sample volume is $1 \mu \mathrm{L}$ and the deposition area is about $1.7 \times 1.7 \mathrm{~mm}^{2}$. (b) Loglog plot of the counts versus the molar concentration of the AuNR probe solution. The sample volume is $1 \mu \mathrm{L}$. Error bars are the standard deviation over three replicate measurements. 
higher concentrations result in significant electrostatic repulsion and counting errors due to the small distance between two adjacent AuNRs, which reduces the quantification accuracy to some extent. The level of low-attomolar detection is the foundation for accurate counting of the target PSA, which has a $1: 1$ ratio in quantity in principle.

\section{Application in PSA detection}

PSA concentrations were detected by counting the confined AuNR probes in dark field images, where each AuNR corresponds to a PSA molecule released from the surface of an MNP. Typical subimages of the released AuNR probes at different concentrations of PSA are shown in Fig. 5. These counts have a good positive correlation with the target concentration (Fig. 6). Using the blank samples, the average of the blank signal was $\sim 115$ and the standard deviation was $\sim 23$. This gave an original LOD of $6 \mathrm{fg} \mathrm{mL}^{-1}$ (however, this may not be theoretically correct). We also performed experiments near the LOD of $\sim 6 \mathrm{fg}$ $\mathrm{mL}^{-1}$ and found the standard deviation to be $\sim 48$, which is much higher than the results from the blank samples. Using the standard formula of LOD $=3 \times$ standard deviation of the signal, the blank signal plus the 3 -fold standard deviation (115+ $48 \times 3=259$ ) was plugged into the fitting equation of $\log y=$ $0.806 \log x+4.098$, giving a final LOD of $\sim 8 \mathrm{fg} \mathrm{mL}^{-1}$. This is roughly equal to the results from a recent study that was also based on individual AuNRs ${ }^{45}$ and is at least $10^{4}$ times higher than a recent study based on single-countable gold nanoparticles using dark field microscopy. ${ }^{\mathbf{4 6}}$ Remarkably, the fitting coefficient in the log-log scale is 0.806 , which is slightly off the linear relationship. This nonlinear relationship may be ascribed to several factors: the relatively high level of background signal,
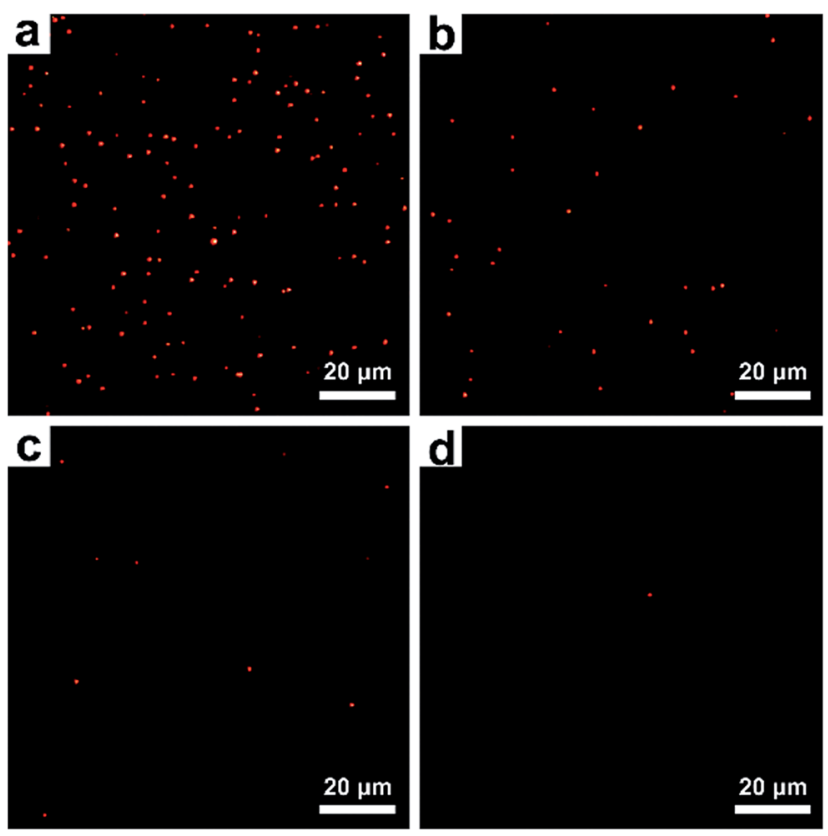

Fig. 5 Typical dark field images of AuNRs released from the MNP surface at various PSA concentrations. (a) $100 \mathrm{pg} \mathrm{mL}^{-1}$, (b) $10 \mathrm{pg} \mathrm{mL}^{-1}$, (c) $1 \mathrm{pg} \mathrm{mL}^{-1}$, and (d) $0.1 \mathrm{pg} \mathrm{mL}^{-1}$.

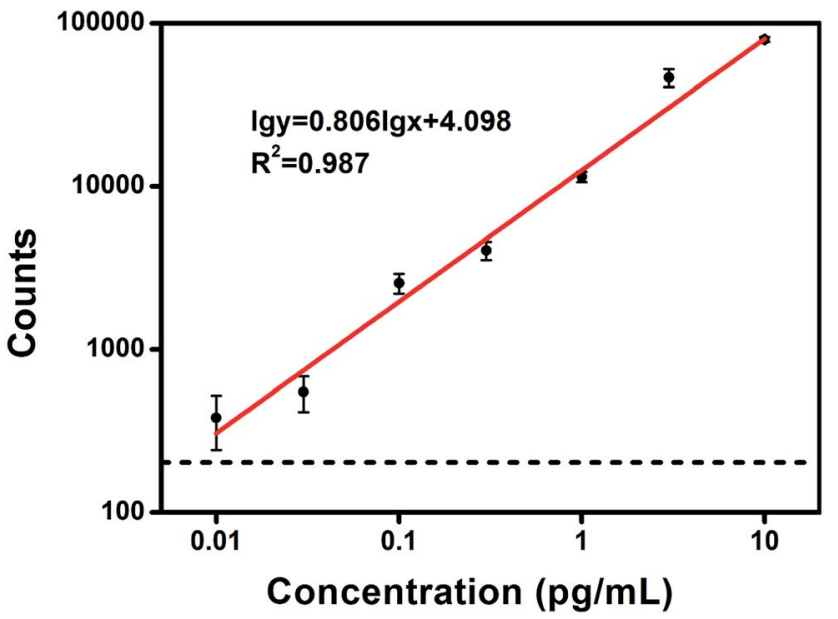

Fig. 6 The standard calibration curve for the detection of PSA, shown in log-log scale (the raw data is shown in Table S1 $\uparrow$ ). The broken line indicates the average of the zero background plus the 3-fold standard deviation of the signal near the LOD. Error bars are the standard deviation over three replicate measurements.

leading to significant errors at low concentrations of PSA; the inefficient probes on the surfaces of magnetic nanoparticles or AuNRs, leading to a low transformation rate at high concentrations of PSA; and the nonlinear isotherms in the target binding kinetics. The linear dynamic range of this method spanned approximately 3 orders of magnitude, ranging from 0.01 to $10 \mathrm{pg} \mathrm{mL}^{-1}$, and the dose response curve is nearly linear within this range. The intensity of the signal increases $\sim 400$ fold when the target concentration increases 1000-fold. This is a distinct advantage over the highly sensitive methods based on signal amplification or target amplification, such as ELISA, scanometric assay ${ }^{47}$ and bio-barcode. ${ }^{48,49}$ With these methods, the signal changes are less than 10-fold when the target concentration increases 1000 -fold or higher within the linear dynamic range. Remarkably, the much broader dose response curve in our method is favorable to reduce uncertainties from the detection instruments and ensures higher reproducibility.

\section{Conclusions}

In summary, we have reported a promising, highly sensitive digital immunoassay. As demonstrated above, the method proposed in this paper represents an advance in the field of analytical chemistry for three reasons. First, magnetic enrichment of the targets and electrostatic confinement of the single molecule probes ensures relatively high detection efficiency. As a result, a detection limit as low as $8 \mathrm{fg} \mathrm{mL}^{-1}$ for the detection of PSA was achieved in a simple manner without the requirement of target or signal amplification procedures, which are universal strategies to improve detection sensitivity. Second, we show that the proposed method has a wide and linear dose response curve over 3 orders of magnitude of the target concentration due to its digital quantification properties. Third, the utilization of AuNRs with extremely strong scattering enables the realization of digital quantification of the target with instrumentation costs 
far below those of SMD methods based on fluorescence detection. In the present form of this digital immunoassay, several problems also exist. The background signal is slightly high, which may be ascribed to non-specific interactions between the MNPs and AuNRs. The target binding kinetics appear to not be very effective, which may be ascribed to the immature probe preparation process. The liquid operation is labor-intensive; this may be optimized by the introduction of microfluidic chips. In addition, the repetition of the detection results should be further improved for more reliable results. It is believed that this method has the potential to facilitate earlier clinical diagnosis in the near future.

\section{Acknowledgements}

This work was supported by the National Science Foundation of China (grants 21273126, 61675113 and 21573124) and the Fundamental Research Program of Shenzhen (JCYJ20140509172959966, JCYJ20160317152359560). HT is grateful for financial support from The Science Technology Innovation Commission of Shenzhen Municipality (GJHZ20160301163644983) and The Health and Family Planning Commission of Shenzhen Municipality (201601019).

\section{Notes and references}

1 J. L. Khatcheressian, P. Hurley, E. Bantug, L. J. Esserman, E. Grunfeld, F. Halberg, A. Hantel, N. L. Henry, H. B. Muss and T. J. Smith, J. Clin. Oncol., 2013, 31, 961-965.

2 J. Mok, M. N. Mindrinos, R. W. Davis and M. Javanmard, Proc. Natl. Acad. Sci. U. S. A., 2014, 111, 2110-2115.

3 S. Howorka and J. Hesse, Soft Matter, 2014, 10, 931-941.

4 M. Cretich, G. G. Daaboul, L. Sola, Ü. M. Selim and M. Chiari, Trends Biotechnol., 2015, 33, 343-351.

5 M. Kühnemund and M. Nilsson, Biosens. Bioelectron., 2014, 67, 11-17.

6 C. Ma and E. S. Yeung, Anal. Bioanal. Chem., 2010, 397, 22792284.

7 G. Li, L. Zhu, Z. Wu, Y. He, H. Tan and S. Sun, Anal. Chem., 2016, 88, 10994-11000.

8 J. Todd, B. Freese, A. Lu, D. Held, J. Morey, R. Livingston and P. Goix, Clin. Chem., 2007, 53, 1990-1995.

9 L. Li, X. Tian, G. Zou, Z. Shi, X. Zhang and W. Jin, Anal. Chem., 2008, 80, 3999-4006.

10 L. Li, X. Wang, X. Zhang, J. Wang and W. Jin, Anal. Chim. Acta, 2015, 854, 122-128.

11 J. Hesse, J. Jacak, M. Kasper, G. Regl, T. Eichberger, M. Winklmayr, F. Aberger, M. Sonnleitner, R. Schlapak and S. Howorka, Genome Res., 2006, 16, 1041-1045.

12 J. Todd, B. Freese, A. Lu, D. Held, J. Morey, R. Livingston and P. Goix, Clin. Chem., 2007, 53, 1990-1995.

13 Z. Wang, Q. Xue, W. Tian, L. Wang and W. Jiang, Chem. Commun., 2012, 48, 9661-9663.

14 J. Zhou, Q. X. Wang and C. Y. Zhang, J. Am. Chem. Soc., 2013, 135, 2056-2059.

15 A. Agrawal, C. Zhang, T. Byassee, R. A. Tripp and S. Nie, Anal. Chem., 2006, 78, 1061-1070.
16 X. Zhao, R. Tapec-Dytioco and W. Tan, J. Am. Chem. Soc., 2003, 125, 11474-11475.

17 Q. Xue, D. Jiang, L. Wang and W. Jiang, Bioconjugate Chem., 2010, 21, 1987-1993.

18 U. Reschgenger, M. Grabolle, S. Cavalierejaricot, R. Nitschke and T. Nann, Nat. Methods, 2008, 5, 763-775.

19 P. S. Dittrich and P. Schwille, Appl. Phys. B, 2001, 73, 829837.

20 J. Azoulay, A. Débarre, A. Richard and P. Tchénio, Phys. Rev. Lett., 2006, 96, 374-380.

21 X. Michalet, F. F. Pinaud, L. A. Bentolila, J. M. Tsay, S. Doose, J. J. Li, G. Sundaresan, A. M. Wu, S. S. Gambhir and S. Weiss, Science, 2005, 307, 538-544.

22 C. Burda, X. Chen, R. Narayanan and M. A. El-Sayed, Chem. Rev., 2005, 105, 1025-1102.

23 P. K. Jain, K. S. Lee, I. H. Elsayed and M. A. Elsayed, J. Phys. Chem. B, 2006, 110, 7238-7248.

24 L. Tong, Q. Wei, A. Wei and J. X. Cheng, Photochem. Photobiol., 2009, 85, 21-32.

25 X. H. Huang, I. H. EI-Sayed, W. Qian and M. A. El-sayed, J. Am. Chem. Soc., 2006, 128, 2115-2120.

26 S. Sun, M. Gao, G. Lei, H. Zou, J. Ma and C. Huang, Nano Res., 2016, 9, 1125-1134.

27 S. Berensmeier, Appl. Microbiol. Biotechnol., 2006, 73, 495504.

28 R. S. Molday and D. Mackenzie, J. Immunol. Methods, 1982, 52, 353-367.

29 A. R. Reeves, K. L. Spiller, D. O. Freytes, G. Vunjak-Novakovic and D. L. Kaplan, Biomaterials, 2015, 73, 272-283.

30 F. M. Kievit, Z. R. Stephen, K. Wang, C. J. Dayringer, J. G. Sham, R. G. Ellenbogen, J. R. Silber and M. Zhang, Mol. Oncol., 2015, 9, 1071-1080.

31 K. Won, M. J. Park, H. H. Yoon and J. H. Kim, Ultramicroscopy, 2008, 108, 1342-1347.

32 S. Link and M. A. El-Sayed, Annu. Rev. Phys. Chem., 2003, 54, 331-366.

33 I. C. Pekcevik, L. C. Poon, M. C. Wang and B. D. Gates, Anal. Chem., 2013, 85, 9960-9967.

34 C. Wang and J. Irudayaraj, Small, 2008, 4, 2204-2208.

35 A. Wijaya and K. Hamad-Schifferli, Langmuir, 2008, 24, 99669969.

36 J. J. Storhoff, A. A. Lazarides, R. C. Mucic, C. A. Mirkin, R. L. Letsinger and G. C. Schatz, J. Am. Chem. Soc., 2000, 122, 4640-4650.

37 J. J. Storhoff, R. Elghanian, R. C. Mucic, C. A. Mirkin and R. L. Letsinger, J. Am. Chem. Soc., 1998, 120, 1959-1964.

38 H. Jans, X. Liu, L. Austin, G. Maes and Q. Huo, Anal. Chem., 2009, 81, 9425-9432.

39 L. Tang, S. Li, F. Han, L. Liu, L. Xu, W. Ma, H. Kuang, A. Li, L. Wang and C. Xu, Biosens. Bioelectron., 2015, 71, 7-12.

$40 \mathrm{~J}$. Wu, W. Jia, W. Lu and L. Jiang, ACS Appl. Mater. Interfaces, 2012, 4, 6560-6564.

41 L. Xie, X. Yan and Y. Du, Biosens. Bioelectron., 2014, 53, 5864.

42 N. Nath and A. Chilkoti, Anal. Chem., 2002, 74, 504-509. 
43 K. M. Mayer, S. Lee, H. Liao, B. C. Rostro, A. Fuentes, P. T. Scully, C. L. Nehl and J. H. Hafner, ACS Nano, 2008, 2, 687-692.

44 J. Y. Byun, Y. B. Shin, T. Li, J. H. Park, D. M. Kim, D. H. Choi and M. G. Kim, Chem. Commun., 2013, 49, 9497-9499.

45 P. L. Truong, C. Cao, S. Park, M. Kim and S. J. Sim, Lab Chip, 2011, 11, 2591-2597.

46 C. Y. Poon, L. Wei, Y. Xu, B. Chen, L. Xiao and H. W. Li, Anal. Chem., 2016, 88, 8849-8856.
47 W. Cheng, Y. Chen, F. Yan, L. Ding, S. Ding, H. Ju and Y. Yin, Chem. Commun., 2011, 47, 2877-2879.

48 B. K. Oh, J. M. Nam, S. W. Lee and C. A. Mirkin, Small, 2006, 2, 103-108.

49 C. S. Thaxton, R. Elghanian, A. D. Thomas, S. I. Stoeva, J. S. Lee, N. D. Smith, A. J. Schaeffer, H. Klocker, W. Horninger and G. Bartsch, Proc. Natl. Acad. Sci. U. S. A., 2009, 106, 18437-18442. 